\title{
A NEW PROPER MOTION SURVEY OF THE PLEIADES CLUSTER
}

\author{
E. SCHILBAC'H ${ }^{1}$, J. GUIBERT ${ }^{2}$, M. GEFFERT ${ }^{3}$, S. HIRTE $^{1}$ \\ ${ }^{1}$ Universität Potsdam. Sternwarte Babelsberg \\ An der Sternwarte, 16 \\ O-1590 Potsdam, Germany \\ ${ }^{2}$ Centre d'Analyse des Images/MAMA, Observatoire de Paris \\ 61 av. de l'Observatoire \\ 75014 Paris, France \\ ${ }^{3}$ Observatorium Hoher List \\ W-5.568 Daun, Germany
}

\begin{abstract}
A programme for the determination of proper motions and photographic B,V.R magnitudes for stars up to $18^{m}$ within a $4^{\circ}$ by $4^{\circ}$ region centered near Alcyone is described. We use MAMA measurements of plates taken with Tautenburg and OCA Schmidt telescopes as well as with the double refractor of Bonn and Carte du Ciel plates. To check the stability of the solution three different methods of reduction are applied.

According to the results of the pilot programme a final proper motion accuracy of about 2 mas/a can be achieved for the majority of stars in the survey.
\end{abstract}

\section{Introduction}

For our understanding of star formation in the Galaxy as well as for a test of dynamical models of galactic clusters it is important to improve the database of proper motions and photometry of stars in the field of open clusters. Deep and accurate proper motion surveys supplemented by photometry provide effective data to identify cluster members and herewith to improve the basis for theoretical interpretation.

With a distance of $130 \mathrm{pc}$ from the Sun, the Pleiades are one of the nearest open clusters. Its remarkable feature and the high density of bright stars within a small field led to the fact that numerous photographic plates of the cluster were already obtained 50 to 100 years ago. The combination of these observations with Schmidt plates taken within the last decades provides a valuable source of accurate proper motion determinations for stars over a wide range of magnitudes.

During last years much effort has been undertaken to identify faint cluster members of the Pleiades (e.g. Stauffer et al. 1991, Hambly et al. 1992). Nevertheless, precise proper motion studies have been mostly restricted to the central field of $1.5^{\circ} \times 1.5^{\circ}$ and to a limiting magnitude of $\mathrm{m}=12$ (Jones 1970, Vasilevskis et al. 1979). Analysing the internal 
motions in the Pleiades Jones (1970) found a velocity anisotropy of the cluster. Later, considering the same data McNamara et al. (1986) supposed that the anisotropy claimed to exist within the Pleiades might be caused by some data and/or reduction effects. It is therefore very relevant to reinvestigate a velocity anisotropy in the Pleiades with precise data from a larger field.

We are performing a proper motion survey and a photographic $\mathrm{B}, \mathrm{V}, \mathrm{R}$ photometry up to 18 magnitude covering a 16 square degree region centered near Alcyone. For this purpose we use plates taken with the Schmidt telescopes of the Tautenburg Observatory and Observatoire de la Côte d'Azur (OC'A) as well as with the double refractor of Bonn and plates of different Carte du Ciel zones. We will be able to achieve an accuracy of $0.15 \mathrm{mag}$ and 2 milliarcseconds/year (mas/a) for photometric data and proper motions, respectively. In the central field of $1.5^{\circ} \times 1.5^{\circ}$, the accuracy of the proper motions may be improved to $0.6 \mathrm{mas} / \mathrm{a}$ for stars brighter than $14 \mathrm{mag}$. Results of the pilot programme are given in Section 4.

The main goals for our survey are:

- reliable determination of the Pleiades membership by use of astrometric and photometric criteria and improving the luminosity function up to the 18 magnitude; comparision with recent results by Hambly et al. (1992) and Stauffer et al. (1991).

- setting dynamical constraints to models of galactic clusters by the determination of velocity dispersion and velocity anisotropy, especially in the outer parts of the Pleiades. The data will also enable a better mass estimation of the cluster.

As a by-product, proper motions and colours of field stars can be used for a test of galactic models (Bienaymé et al. 1992, Soubiran 1992), and a catalogue of accurate positions and proper motions can provide a basis for astrometric tests of telescopes.

For the study of global features in the proper motions we intend to use later HIPPARCOS data because the small number of galaxies in the examined field will not provide a reliable link to the extragalactic reference system.

\section{Observations, Plate Measurements, and Plate Matching}

An essential part of observations for the proper motion survey is based on plates taken with the Tautenburg Schmidt telescope $(134 / 203 / 401)$. 12 partly overlapping plate pairs cover a total field of about 18 square degrees. A central field of 2.6 square degrees is overlapped by all plates. The remaining field is overlapped on average by 12 plates, with the exception of a small part of about 3 square degrees covered only by one of the plate pairs. The epoch difference for the plate pairs ranges from 26 to 30 years and the limiting magnitude from $18.5 \mathrm{mag}$. to $20 \mathrm{mag}$.

In addition, for the central field about 50 plates taken with the double refractor ( $\mathrm{D}=30 \mathrm{~cm}, \mathrm{~F}=500 \mathrm{~cm}$ ) at Bonn from 1901 to 1992 as well as 15 Carte du Ciel plates (Paris, Bordeaux and Algiers zones) from 1902 to 1942 are included in the programme.

For the photographic $B, V, R$ photometry at least two plates in each colour taken with the OCA Schmidt telescope $(90 / 152 / 316)$ are used. With a size of $5.2^{\circ} \times 5.2^{\circ}$ an $\mathrm{OC}$ A 
plate covers the complete investigated field. Therefore, the measurements of the deep OCA plates are also used for compiling a reference list providing a cross-identification between stars measured on different plates.

All plates are scanned with the Machine Automatique à Mesurer pour l'Astronomie (MAMA) in its systematic mode with a pixel size of $10 \mu \mathrm{m}$ (Berger et al. 1991). Additionally, astrometric (PPM-stars) and photometric (Stauffer 1984) standards are measured in pavé - mode.

The matching routine consists in pairing up of objects between the reference plate and a comparison plate starting with bright stars measured in pave mode. A target search radius is fixed in relation with the scales and with the epoch difference of the plates as a compromise between losing high proper motion stars and an ambiguous or false identification. An additional criterion based on the comparison of measured stellar brightness is applied.

\section{Astrometric Reduction of Measurements}

To improve the systematic accuracy of proper motions we intend to apply three different reduction techniques. The final reduction method will be chosen after analysing the results obtained with these different techniques. Additionally, we will estimate how different reduction techniques may influence the final results.

\subsection{PLATE - TO - PLATE SOLUTION BY USE OF ORTHOGONAL POLYNOMIALS}

Each plate is iteratively transformed to the reference plate by use of Legendre polynomials up to the 4th order (Soubiran 1992). We start with all stars having a counterpart on the reference plate and reject from the consecutive solution those stars with residuals more than twice the value of rms. The procedure stops when the number of rejected stars in the iterative step is lower than 0.5 per cent of the original number of stars. Finally, the proper motion for each star is obtained from corresponding residuals in dependence on a plate epoch by a least square solution.

\subsection{ITERATIVE METHOD BY USE OF A REFERENCE CATALOGUE}

In a first step a classical determination of positions of all stars on each plate is carried out with respect to a set of catalogue stars with known positions and proper motions. Positions and proper motions of each star are then obtained by a corresponding least square adjustment of the determined positions in dependence on the observational epochs. In the next steps the measurements are always reduced to the "catalogue" obtained in the solutions before. As a rule, after the 3 th to 5 th iteration positions and proper motions do no longer show significant changes.

\subsection{OVERLAPPING TECHNIQUE BY EICHHORN (1960)}

The overlapping method takes into account the fact that a star appearing on different 
plates taken in the same epoch must have the same position. Therefore, the equations for all stars on all plates are put together into one system, where plate constants as well as positions and proper motions are unknown. The corresponding coefficient matrix is rather large but very sparse (only about 0.017 per cent nonzero elements). To carry out a direct solution we choose the special storage technique, sparse row-wise format (Pissanetzky, 1984 ) and the algorithm developed by Peters and Wilkinson (1970). This algorithm does not use the ill-conditioned normal matrix but a special LU-decomposition of the coefficient matrix.

\section{Results of the Pilot Programme}

To estimate what accuracy of proper motions can be achieved we used MAMA measurements of one OCA plate and 8 Tautenburg programme plates (Table 1). Each Tautenburg plate was matched with the OCA plate. Cross-identification between the same objects measured on different Tautenburg plates was carried out by use of OCA reference number. For each pair of Tautenburg plates, proper motions were derived with the reduction method described in 3.1. Generally, 10 to 15 iterations were carried out. The least square residuals (rms) in right asscension (RA) and in declination (DE) are given in Table 2.

Table 1. Plate material

\begin{tabular}{cccc}
$\begin{array}{c}\text { Plate No. } \\
\text { (pairs) }\end{array}$ & Epoch & Colour & $\begin{array}{c}\text { Plate centre } \\
(1950.0)\end{array}$ \\
T549/T7818 & $1962.74 / 1991.95$ & $\mathrm{~V}$ & $3^{h} 44.0^{m}+24^{\circ} 19^{\prime}$ \\
T550/T7809 & $1962.74 / 1991.94$ & $\mathrm{~B}$ & $3^{h} 44.0^{m}+24^{\circ} 19^{\prime}$ \\
T553/T7816 & $1962.74 / 1991.95$ & $\mathrm{~V}$ & $3^{h} 44.0^{m}+24^{\circ} 19^{\prime}$ \\
T624/T7828 & $1962.76 / 1991.96$ & $\mathrm{~B}$ & $3^{h} 43.9^{m}+24^{\circ} 38^{\prime}$ \\
OCA462 & 1981.10 & $\mathrm{R}$ & $3^{h} 45.3^{m}+23^{\circ} 57^{\prime}$ \\
\hline
\end{tabular}

Table 2. Plate - to - plate solution

\begin{tabular}{cccc} 
Plate pairs & $\begin{array}{c}\text { Number of } \\
\text { common stars }\end{array}$ & $\begin{array}{c}\text { Number of } \\
\text { reference stars }\end{array}$ & $\begin{array}{c}\text { rms ( arcsec ) } \\
\text { RA/DE }\end{array}$ \\
T549/T7818 & 9275 & 4446 & $0.13 / 0.14$ \\
T550/T7809 & 15782 & 7194 & $0.12 / 0.14$ \\
T553/T7816 & 14189 & 6579 & $0.12 / 0.15$ \\
T624/T7809 & 21624 & 10127 & $0.11 / 0.11$ \\
\hline
\end{tabular}

After the elimination of objects falsely matched we obtained a list of stars with 1 to 4 values for their proper motions. From these values we estimated an average accuracy of $6.3 \mathrm{mas} / \mathrm{a}, 4.3 \mathrm{mas} / \mathrm{a}$ and $3.1 \mathrm{mas} / \mathrm{a}$ for proper motions derived from measurements of 2,3 and 4 plate pairs, respectively. The rms of the mean proper motions of stars measured on at least 3 plate pairs in dependence on their decimal logarithms of MAMA density fluxes are given in Table 3. The density flux of a star image is the internal brightness estimation 
of the star which can be converted into stellar magnitudes. The corresponding magnitudes range from $18 \mathrm{mag}$ to $6.5 \mathrm{mag}$.

Table 3. Proper motion accuracy of stars measured on at least 3 plate pairs in dependence on their MAMA density fluxes

\begin{tabular}{|c|c|c|c|c|}
\hline $\lg (F \operatorname{lu} x)$ & $\overline{\lg (F l u x)}$ & $\begin{array}{c}\text { number of } \\
\text { stars }\end{array}$ & $\begin{array}{c}\operatorname{rms}(\mathrm{RA}) \\
(\mathrm{mas} / \mathrm{a})\end{array}$ & $\begin{array}{c}\operatorname{rms}(\mathrm{DE}) \\
(\mathrm{mas} / \mathrm{a})\end{array}$ \\
\hline$<4.15$ & 4.062 & 402 & 5.4 & 5.9 \\
\hline $4.15-4.45$ & 4.323 & 1617 & 4.4 & 5.0 \\
\hline $4.45-4.75$ & 4.615 & 2703 & 3.8 & 4.5 \\
\hline $4.75-5.05$ & 4.896 & 3175 & 2.4 & 3.8 \\
\hline $5.05-5.35$ & 5.180 & 2063 & 1.6 & 3.5 \\
\hline $5.35-5.65$ & 5.418 & 819 & 2.3 & 3.6 \\
\hline $5.65-5.95$ & 5.779 & 251 & 3.2 & 3.6 \\
\hline$>6.10$ & 6.152 & 124 & 4.0 & 4.2 \\
\hline
\end{tabular}

No systematic differences between proper motions, derived from measurements of Band V-plates were found. On the other hand, we can see (Table 3) that proper motion accuracy in RA is slightly higher than in DE. This effect seems to be caused by the measuring procedure, but we cannot explain it unambiguously. Further investigations are required.

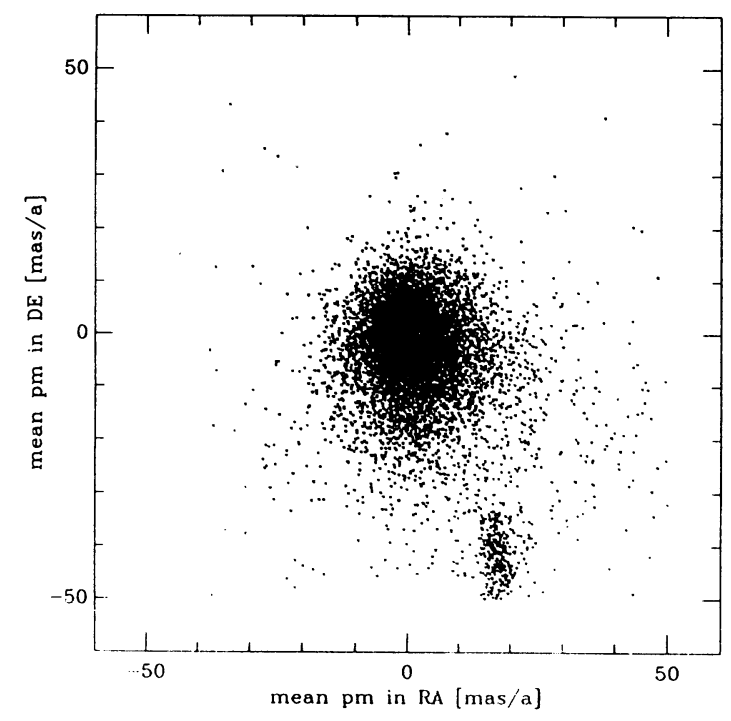

Figure 1. Proper motion vector point diagram for stars measured on at least 3 plate pairs

Figure 1 shows the proper motion vector point diagram for stars measured on 3 or 4 plate pairs where 51 high velocity stars with absolute values of proper motions above 75 mas/a are omitted. Considering only proper motion criteria there are 342 cluster candida- 
tes inside the window from +10 to +25 mas/a in RA and from -55 to -30 mas/a in DE. The velocity of the centroid for this group of +17.5 mas $/ a$ (RA) and -40.9 mas/a (DE) is more than 8 times larger than rms of proper motions. Additionally, according to their values of proper motions, 55 stars measured on two plate pairs and 111 stars measured on only one plate pair can also be considered as possible cluster candidates. Generally, these stars are fainter and extend to the $19 \mathrm{mag}$. The cluster candidates are distributed on the total field of $2.9^{\circ}(\mathrm{RA}) \times 3.3^{\circ}(\mathrm{DE})$ examined.

\section{Conclusions}

An accuracy of about 3 mas/a can be achieved for the proper motions of stars over a wide range of magnitudes, provided that at least 4 plate pairs are used. As an average 12 Tautenburg plates are at our disposal, we expect that for the majority of stars in the survey on accuracy of about 2 mas/a can be obtained. For stars with intermediate magnitudes $(11<m<16)$ the proper motion accuracy may be even higher.

More sophisticated reduction techniques may yield further improvements of the data reliability. Additional tests are to be involved in the programme to investigate the origin for the different accuracies in $\mathrm{x}$ - and $\mathrm{y}$ - directions.

\section{ACKNOWLEDGEMENTS}

We thank the Tautenburg and OCA Observatories for supplying and the MAMA team for measuring the plates. The authors thank Dr. Caroline Soubiran for her plate-to-plate solution computer program. Financial support from PROCOPE (Programme de Cooperation Scientifique between France and Germany) is gratefully acknowledged.

\section{REFERENC'ES}

Berger, J., Cordoni, J.P., Fringant, A.M., Guibert, J., Moreau, O., Reboul, H., Vanderriest, C.: 1991, Astron.Astrophys.Suppl. 87, 389.

Bienaymé, O., Mohan, V., C'rézé, M., Considère, S., Robin, A.C.: 1992, Astron.Astrophys. 253, 389.

Eichhorn, H.: 1960, Astron.Nachr. 285, 16.

Hambly, N.C., Hawkins, M.R.S., Jameson, R.F.: 1992, Mon.Not.R.Astron.Soc. 253, 1.

Jones, B.F.: 1970, Astron.J. 75, 563.

McNamara, B.J., Sekiguchi, K.: 1986, Astrophys.J. 310, 613.

Peters, G., Wilkinson, J.H.: 1970, Computer J. 13, 309.

Pissanetzky, S.: 1984, Sparse Matrix Technology, Academic Press.

Soubiran, C.: 1992, Astron.Astrophys. 259, 394.

Stauffer, J.: 1984, Astrophys.J. 280, 189.

Stauffer, J., Klemola, A., Prosser, C., Probst, R.: 1991, Astron.J. 101, 980.

Vasilevskis, S., van Leeuwen, F., Nicholson, W., Murray, C.A.: 1979, Astron.Astrophys.Suppl. 37, 333. 